\title{
Nitric Oxide Reduces Vagal Baroreflex Sensitivity in the Late Gestation Fetus
}

\author{
AVNESH S. THAKOR, AND DINO A. GIUSSANI \\ Department of Physiology, Development and Neuroscience, University of Cambridge, Cambridge CB2 3EG, United Kingdom
}

\begin{abstract}
Goals to understand the etiology of essential hypertension have proposed that this problem arises, in part, because of changes within brainstem circuits involved in arterial blood pressure (ABP) control. It has been suggested that nitric oxide (NO) exerts inhibitory influences on the integration of afferent discharge from the arterial baroreceptors. This study tested the hypothesis that the inhibitory influence of NO on the arterial baroreflex is present in fetal life. Fetal baroreflex sensitivity was calculated in fetal sheep, before and during the NO-clamp; a technique that permits NO synthase (NOS) blockade with L-NAME while maintaining basal cardiovascular function with sodium nitroprusside. Under halothane anesthesia, five fetal sheep at 0.8 gestation were instrumented with vascular catheters. Five days later, fetuses received a range of bolus doses of phenylephrine (5-75 $\mu \mathrm{g}$ I.A.) in randomized order either during saline or treatment with the NO clamp. Basal fetal ABP and heart rate before $(50 \pm 4 \mathrm{~mm} \mathrm{Hg}, 170 \pm 3 \mathrm{bpm})$ or during $(51 \pm 4 \mathrm{~mm} \mathrm{Hg}$, $173 \pm 3 \mathrm{bpm}$ ) the NO-clamp were similar. The gradient of the pulse interval-ABP relationship was nearly doubled during NOS blockade (14.2 \pm 2.5 versus $7.8 \pm 1.6 \mathrm{~ms} / \mathrm{mm} \mathrm{Hg}$ ). The data provide in vivo evidence that NO attenuates the sensitivity of the cardiac baroreflex during fetal life. (Pediatr Res 65: 269-273, 2009)
\end{abstract}

$\mathrm{T}$ he arterial baroreflex plays an important role in maintaining cardiovascular homeostasis by continuously eliciting autonomic adjustments to maintain arterial blood pressure (ABP) within a narrow physiologic range. Baroreceptors are located in the carotid sinus and aortic arch, where they sense beat-by-beat changes in ABP. This information is relayed via primary afferent neurons to the brainstem, where the signal is processed and integrated. Compensatory adjustments in heart rate (HR) and vascular resistance, for any perturbations in $\mathrm{ABP}$, are subsequently mediated via changes in efferent vagal and sympathetic outflow $(1,2)$.

Studies in both the adult and fetus have shown that there is an age-dependent fall in arterial baroreflex sensitivity $(3,4)$. Accumulating evidence implicates nitric oxide (NO) in baroreflex pathways and its involvement in altering baroreflex sensitivity with ageing. For instance, NO synthase (NOS) immunoreactivity has been demonstrated in several locations

Received June 9, 2008; accepted September 23, 2008.

Correspondence: Dino A. Giussani, Ph.D., Department of Physiology, Development and Neuroscience, University of Cambridge, Cambridge, CB2 3EG, UK; e-mail: dag26@cam.ac.uk

Supported by the "International Journal of Experimental Pathology" and the "Lister Institute for Preventive Medicine." of the baroreflex pathway, including the carotid sinus (5), the nucleus tractus solitarius (6), and the cardiac vagal and preganglionic sympathetic neurons $(7,8)$. Further, the fall in baroreflex sensitivity with advancing age in conscious lambs is dependent on NO (9). However, it remains completely unknown whether the inhibitory influence on baroreflex sensitivity by NO is already present in fetal life. The present study tested the hypothesis that endogenous NO depresses baroreflex sensitivity during fetal life. Our own pilot studies and investigations by Schroder et al. (10) have shown that in fetal life, unloading of the arterial baroreceptors, for instance using bolus doses of NO donors, does not produce robust increases in fetal HR. This reflects the well-established relative immaturity of sympathetic compared with parasympathetic autonomic control of basal HR during fetal life in late gestation (11). Therefore, this study focused on the vagal dominance of the cardiac baroreflex pathway by loading arterial baroreceptors by means of phenylephrine-induced graded increments in ABP. The hypothesis was tested by investigating in vivo baroreflex function during basal conditions in conscious late gestation fetal sheep, before and during the NO-clamp. The NO-clamp is a technique that permits blockade of de novo synthesis of NO while compensating for the tonic production of the gas, thereby maintaining basal cardiovascular function.

\section{MATERIALS AND METHODS}

Surgical preparation. All procedures were performed under the UK Animals (Scientific Procedures) Act 1986 and were approved by the Ethical Review Committee of the University of Cambridge. Five Welsh Mountain sheep fetuses were surgically instrumented for long-term recording at $123 \pm$ $1 \mathrm{~d}$ of gestation (term is $c a .145 \mathrm{~d}$ ), which, in humans, corresponds to the third trimester of pregnancy. Surgery was performed using strict aseptic conditions as previously described in detail (12). In brief, food, but not water, was withheld from the pregnant ewes for $24 \mathrm{~h}$ before surgery. After induction with $20 \mathrm{mg} / \mathrm{kg}$ i.v. sodium thiopentone (Intraval Sodium; Merial Animal Health Ltd., Rhone Mérieux, Dublin, Ireland), general anesthesia (1.5-2.0\% halothane in $50: 50 \mathrm{O}_{2}: \mathrm{N}_{2} \mathrm{O}$ ) was maintained using positive pressure ventilation. Midline abdominal and uterine incisions were made, the fetal hind limbs were exteriorized, and, on one side, femoral arterial (i.d., $0.86 \mathrm{~mm}$; o.d., $1.52 \mathrm{~mm}$; Critchly Electrical Products, NSW, Australia) and venous (i.d., $0.56 \mathrm{~mm}$; o.d., $0.96 \mathrm{~mm}$ ) catheters were inserted. The catheter tips were advanced carefully to the descending aorta and inferior vena cava, respectively. Another catheter was anchored onto the fetal hind limb for recording of the reference amniotic pressure. The uterine incisions were closed in layers, the dead space of the catheters was filled with heparinized saline (80 i.u. heparin/mL in $0.9 \%$ $\mathrm{NaCl}$ ), and the catheter ends were plugged with sterile brass pins. The catheters were then exteriorized via a keyhole incision in the maternal flank and kept inside a plastic pouch sewn onto the maternal skin. 
Postoperative care. During recovery, ewes were housed in individual pens in rooms with a $12 \mathrm{~h}: 12 \mathrm{~h} /$ light:dark cycle where they had free access to hay and water and were fed concentrates twice daily (100 g sheep nuts no. $6 ; \mathrm{H}$ \& C Beart Ltd., Kings Lynn, UK). Antibiotics were administered daily to the ewe (0.20-0.25 mg/kg i.m. Depocillin; Mycofarm, Cambridge, UK) and fetus i.v. and into the amniotic cavity $(150 \mathrm{mg} / \mathrm{kg}$ Penbritin; SmithKline Beecham Animal Health, Welwyn Garden City, Hertfordshire, UK). The ewes also received $2 \mathrm{~d}$ of postoperative analgesia $(10-20 \mathrm{mg} / \mathrm{kg}$ oral Phenylbutazone; Equipalozone paste, Arnolds Veterinary Products Ltd., Shropshire, UK). Generally, normal feeding patterns were restored within $48 \mathrm{~h}$ of recovery. After $72 \mathrm{~h}$ of postoperative recovery, ewes were transferred to metabolic crates where they were housed for the remainder of the protocol. The arterial, venous, and amniotic catheters were connected to sterile pressure transducers (COBE; Argon Division, Maxim Medical, Athens, Texas) and calibrated mean fetal ABP (corrected for amniotic pressure) and fetal HR (triggered via a tachometer from the pulsatility in the ABP signal) were recorded continually using a computerized data acquisition system (Department of Physiology, Cambridge University, UK). While on the metabolic crates, the patency of the fetal catheters was maintained by a slow continuous infusion of heparinized saline (80 i.u. heparin/mL at $0.1 \mathrm{~mL} / \mathrm{h}$ in $0.9 \% \mathrm{NaCl}$ ) containing antibiotic (1 $\mathrm{mg} / \mathrm{mL}$ benzylpenicillin; Crystapen, Schering-Plough, Animal Health Division, Welwyn Garden City, UK).

Experimental protocol. After at least $5 \mathrm{~d}$ of postoperative recovery, all fetuses received a range of bolus doses of phenylephrine (5-75 $\mu \mathrm{g}$ of L-phenylephrine; P-6126, Sigma Chemical Co. Chemicals, UK) dissolved in $1 \mathrm{~mL}$ of heparinized saline via the femoral artery, in randomized order. Each dose was injected over 2-3 s and was administered after at least $15 \mathrm{~min}$ of stable baseline recording. Doses were first given during a slow i.v. infusion of vehicle (80 i.u. heparin/mL in $0.9 \% \mathrm{NaCl}$ ) and then during NOS blockade using the NO-clamp either the same or the following day. In brief, a bolus dose $\left(100 \mathrm{mg} / \mathrm{kg}\right.$ dissolved in $2 \mathrm{~mL}$ heparinized saline) of L-NAME $\left(N^{\mathrm{G}}\right.$ nitro-L-arginine methyl ester; Sigma Chemical Co. Chemicals, UK) was injected via the femoral artery. This was immediately followed by fetal i.v. infusion with the NO donor, sodium nitroprusside (SNP; Sigma Chemical Co. Chemicals, UK; $5.1 \pm 2.0 \mu \mathrm{g} / \mathrm{kg} / \mathrm{min}$ : mean $\pm 1 \mathrm{~S} . \mathrm{D}$; dissolved in heparinized saline), the concentration of which was titrated to return fetal ABP to basal values in each individual animal. In practice, and with knowledge obtained in previous studies of the dose ranges required, L-NAME and SNP were almost co-administered, such as to avoid any perturbation in basal ABP or basal HR. Although fetal treatment with L-NAME alone leads to pronounced systemic vasoconstriction and hypertension, combined treatment of the fetus with both L-NAME and SNP compensates for the tonic production of the gas, maintains basal cardiovascular function and blocks de novo synthesis of NO during stimulated conditions. This technique has been established in our laboratory and previously validated $(13,14)$. At the end of the experimental protocol, the effectiveness of NOS blockade by the NOclamp and the persistence of L-NAME in the system were tested by withdrawal of the SNP infusion. This unmasked the influence of fetal treatment with L-NAME alone and led to a significant increase in ABP and a fall in HR. During this time, the effects on baroreflex sensitivity of NOS blockade with L-NAME alone in the absence of SNP could also be investigated. At the end of the experimental protocol, the ewes and fetuses were humanely killed using a lethal dose of sodium pentobarbitone $(200 \mathrm{mg} / \mathrm{kg}$ i.v. Pentoject; Animal Ltd., York, UK) and the positions of the implanted catheters were confirmed.

Blood sampling regimen. Before each experimental protocol, descending aortic blood samples $(0.3 \mathrm{~mL})$ were taken using sterile techniques from the fetus to determine arterial blood gas and acid base status (ABL5 Blood Gas Analyzer, Radiometer; Copenhagen, Denmark; measurements corrected to $39.5^{\circ} \mathrm{C}$ ). Values for percentage saturation of $\mathrm{Hb}$ with oxygen (Sat $\mathrm{Hb}$ ) and the blood $\mathrm{Hb}$ concentration $(\mathrm{Hb})$ were determined using a hemoximeter (OSM3; Radiometer). In addition, blood glucose and lactate concentrations were measured by an automated analyzer (Yellow Springs 2300 Stat Plus Glucose/ Lactate Analyzer; YSI Ltd., Farnborough, UK).

Data and statistical analyses. Values for all blood gas variables are expressed as mean \pm SEM. Baseline values for ABP and HR were obtained by averaging the data over the $60 \mathrm{~s}$ preceding the administration of each bolus dose of phenylephrine. HR and blood pressure values were matched on a beat-by-beat basis. Baroreflex curves were constructed for each fetus before and during NOS blockade by plotting for each dose, the maximal increase in $\mathrm{ABP}$ against the corresponding change in pulse interval (PI). PI values were calculated as the reciprocal of corresponding HR values. Correlation analysis of the relationship between blood pressure and PI in all individual fetuses was assessed using the Pearson Product Moment correlation, with the gradient of the PI-ABP relationship representing baroreflex sensitivity $(3,15)$. The mean change in baroreflex sensitivity during the NO-clamp and the mean change in ABP and HR following removal of the NO-clamp, were assessed statistically using the $t$ test for paired data (Sigma Chemical Co. Stat; SPSS Inc., Chicago, IL). For all comparisons, statistical significance was accepted when $p<0.05$.

\section{RESULTS}

Fetal arterial blood gas and metabolic status. Fetal arterial blood gases and metabolic status on the day of the experiment were within the normal range for Welsh Mountain sheep at $c a .125 \mathrm{~d}$ of gestation: $\mathrm{pH}_{\mathrm{a}}=7.35 \pm 0.01$; acid-base excess $(\mathrm{ABE})=0.6 \pm 0.4$ mequiv/L; $\mathrm{HCO}_{3}{ }^{-}=24.4 \pm 1.4$ mequiv $/ \mathrm{L}$;

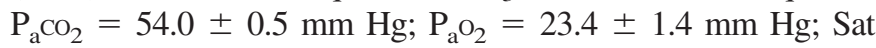
$\mathrm{Hb}=58.3 \pm 2.0 \% ; \mathrm{Hb}=9.4 \pm 0.8 \mathrm{~g} / \mathrm{dL} ;$ lactate $=0.7 \pm 0.1$ $\mathrm{mM}$; and glucose $=0.8 \pm 0.1 \mathrm{mM}$. Neither treatment with phenylephrine or fetal exposure to the NO-clamp affected basal arterial blood gas or metabolic status.

Fetal cardiac baroreflex curves. Fetal ABP $(50 \pm 4 \mathrm{~mm}$ $\mathrm{Hg})$ and $\mathrm{HR}(170 \pm 3 \mathrm{bpm})$ on the day of the experiment were within normal ranges for Welsh Mountain sheep at $c a .128 \mathrm{~d}$ of gestation. The individual baroreflex curves in response to phenylephrine for all fetuses are shown in Fig. 1. For each fetus, correlation analysis between PI and ABP revealed significant relationships. In all fetuses, progressive increments in
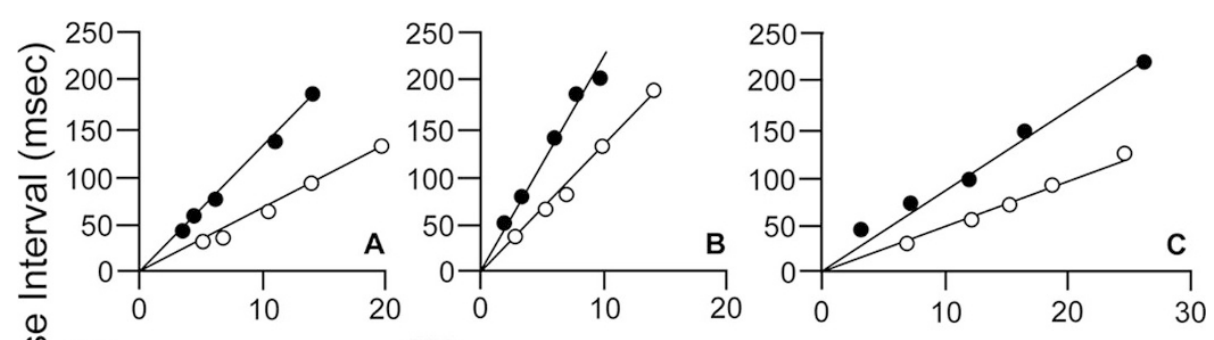

Figure 1. Individual baroreflex curves. Each graph represents the change PI for increases ABP before $(\bigcirc)$ and during $(\bullet)$ NOS blockade with the NO-clamp for five individual fetuses. The Pearson Product Moment correlation coefficients $(r)$ for each linear PI-ABP relationship are: Animal A-O: 0.998, O:0.995; Animal B—O:0.997, ๑:0.992; Animal C-○:0.994, ๑:0.995; Animal D—O:0.998, @:0.996; Animal E-O:0.991, O:0.997.

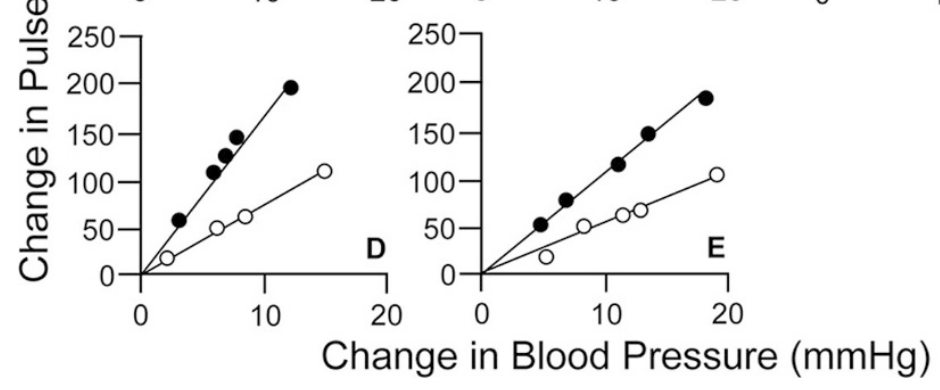



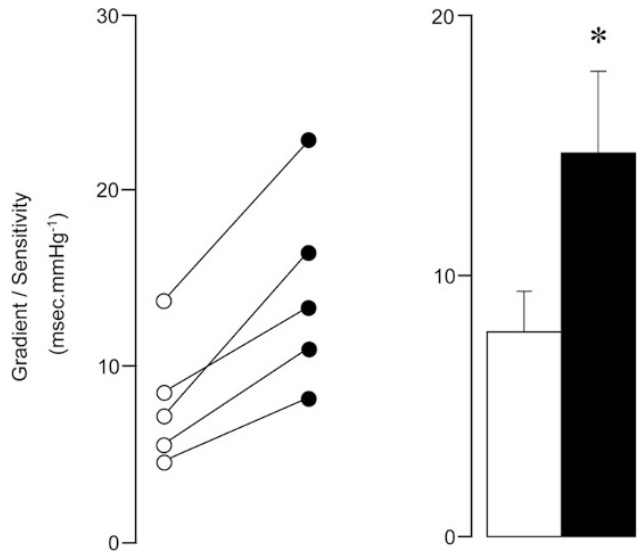

Figure 2. Baroreflex sensitivity before and during the NO-clamp. The data show the individual change in baroreflex sensitivity (PI-ABP gradient) before $(\bigcirc)$ and during $(\bigcirc)$ NOS blockade with the NO-clamp for five individual fetuses. Histograms represent the mean \pm SEM for baroreflex sensitivity (PI-ABP gradient) before ( $\square ; n=5$ ) and during $(\square ; n=5)$ NOS blockade with the NO-clamp. Significant differences: ${ }^{*} p<0.05$, before $v s$ during the NO-clamp ( $t$ test for paired data).
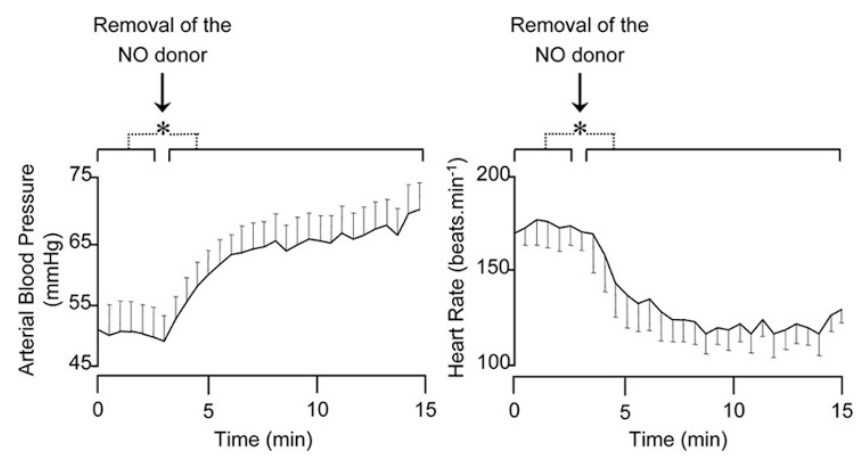

Figure 3. The effect on ABP and HR of removal of the NO donor. Values represent the mean \pm SEM calculated every $30 \mathrm{~s}$ for $\mathrm{ABP}$ and $\mathrm{HR}$ in response to removal of the NO donor (arrow). Significant differences: ${ }^{*} p<0.05$, during $v s$ removal of the NO donor ( $t$ test for paired data).

ABP elicited progressive increments in PI. The change in fetal cardiac baroreflex sensitivity is expressed either individually or as the mean change before and after the NO-clamp (Fig. 2). Fetal ABP and HR before $(50 \pm 4 \mathrm{~mm} \mathrm{Hg}, 170 \pm 3 \mathrm{bpm})$ or during (51 $\pm 4 \mathrm{~mm} \mathrm{Hg}, 173 \pm 3 \mathrm{bpm})$ the NO-clamp were similar, confirming that the blood pressure clamp had worked. During control conditions with saline infusion, the fetal cardiac baroreflex sensitivity was $7.8 \pm 1.6 \mathrm{~ms} / \mathrm{mm} \mathrm{Hg}$. In contrast, the NO-clamp resulted in a significant increase in the fetal cardiac baroreflex sensitivity $(14.2 \pm 2.5 \mathrm{~ms} / \mathrm{mm} \mathrm{Hg}$; $p<0.05$; Fig. $2 B$ ).

Withdrawal of the SNP infusion at the end of the experimental protocols led to a significant increment in fetal ABP $(\Delta 15 \pm 2 \mathrm{~mm} \mathrm{Hg} ; p<0.05)$ and a significant decrement in fetal HR ( $\Delta-65 \pm 12 \mathrm{bpm} ; p<0.05$; Fig. 3). These changes provided evidence for the effectiveness of NOS blockade by the NO-clamp and the persistence of L-NAME within the fetal circulation until the end of the experiment. The changes in ABP and HR after removal of SNP in the presence of L-NAME are also consistent with the idea that persisting NOS blockade in the absence of SNP treatment increased baroreflex sensitivity, as a mean change in $\mathrm{ABP}$ of $12 \pm 2 \mathrm{~mm} \mathrm{Hg}$ coupled with a mean change in PI of $133 \pm 34 \mathrm{~ms}$ falls on the relationships representing baroreflex sensitivity during NOS blockade (filled in circles) rather than during saline (open circles) in all fetuses studied (Fig. 1).

\section{DISCUSSION}

The present study tested the hypothesis that endogenous NO has a physiologic role in fetal life to depress the sensitivity of the cardiac baroreflex. The data show that in the fetus, cardiac baroreflex sensitivity increases during NOS blockade, thereby supporting the hypothesis that increased NO promotes a fall in the sensitivity of the cardiac baroreflex, even before birth.

Much of the information regarding the autonomic control of the arterial baroreflex has been inferred from the effects of HR responses. Chen et al. (16) showed that the immediate fall in $\mathrm{HR}$, which occurred in less than $10 \mathrm{~s}$ in response to arterial baroreceptor loading, was the result of vagal dominance with sympathetic withdrawal. Our results show that in the fetus graded increments in ABP result in progressively larger decrements in fetal HR and corresponding larger increments in fetal PI. Furthermore, during fetal life the sensitivity of the cardiac baroreflex is enhanced during NOS blockade using the NO-clamp. This finding in fetal life is supported by evidence from experimental studies in the adult animal, which have reported an increase in baroreflex sensitivity in the conscious rat (17) and rabbit (18) following systemic administration of NOS inhibitors, such as L-NAME or L-NNA. However, when these NOS inhibitors are given systemically and in isolation, they block both tonic and de novo synthesis of NO, at the expense of altering basal cardiovascular function. Similarly, intracerebroventricular administration of NOS inhibitors to block the central actions of NO is also known to have pronounced effects on basal cardiovascular function (19). To circumvent confounding problems imposed by alterations in basal cardiovascular physiology in the interpretation of data on baroreflex function, the present study used the NO-clamp $(13,14)$. The technique permits blockade of de novo synthesis of NO while compensating for the tonic production of the gas, thereby maintaining basal cardiovascular function. Thus, the technique provides an indispensable and powerful tool to address the effects of de novo synthesis of $\mathrm{NO}$, but not of tonic levels of NO, on baroreflex function in vivo at the organismal level.

In the fetus, the site of action of NO within the baroreflex pathway cannot be inferred from this study but it may be at the level of the sensor, the integrator, and/or the effector organs. In the adult, although NOS immunoreactivity has been detected both in the carotid sinus (5) and in the glossopharyngeal and vagal afferent neurons $(20,21)$, the role of $\mathrm{NO}$ at the level of the sensor of the baroreflex arc remains unclear at any stage of life. However, investigations by Chapleau (22) support that enhanced NO can inhibit baro- and chemoreceptor afferent nerve activity. NO can also influence many regions of the brainstem $(6,23)$, however, the available literature on the actions of $\mathrm{NO}$ at integrator sites is inconsistent with data 
supporting and opposing NO-mediated inhibition of the cardiac baroreflex. For instance, using in vivo unanesthetised decerebrate rats, elegant work from Paton's laboratory has shown that NO is able to act in the caudal NTS to attenuate the gain of the vagal component of the cardiac baroreflex $(24,25)$. This is consistent with other in vivo evidence, which has shown that administration of NOS inhibitors in the NTS increased baroreflex sensitivity in both anesthetized and conscious animals $(26,27)$. Conversely, Chowdhary and Townend (28) have reported that NO serves to inhibit central sympathetic outflow and studies by Di Paola et al. (29) show that the neurotransmitter L-glutamate which promotes the release of NO from nitrergic neurones stimulates, rather than inhibits, baroreflex processing within the NTS. Furthermore, singleneuron recordings within the NTS have shown reduced activity after systemic administration of L-NAME (30) and increased neuronal discharge after NO donors such as L-arginine and SNP (31). NO-mediated inhibition of the cardiac baroreflex appears not to be mediated at the level of the heart itself, since the available literature suggests that NO potentiates, rather than depresses, the magnitude of induced bradycardia (32-34). For example, in vitro and in vivo studies have shown that NOS inhibition blocks the negative cardiac chronotropic effect of muscarinic receptor stimulation in spontaneously beating neonatal rat cardiomyocytes (32) or significantly attenuates the bradycardic response to efferent vagal stimulation (33). Conversely, treatment with NO donors such as molsidomine and SNP significantly enhanced the decrease in HR in response to vagal nerve stimulation in the anesthetized rabbit and isolated guinea pig atria (34).

If NO donors act to facilitate reflex bradycardia (34), it could be argued that the present data represents an artifact of the concomitant administration of SNP during the establishment of the NO-clamp. In addition, it has been reported that SNP has nonspecific pharmacological effects, for instance by promoting the release of iron and cyanide (35) and that iron overload can enhance baroreflex sensitivity (36). However, in the present study, withdrawal of the SNP infusion at the end of the experimental protocols led to a significant increase in fetal ABP and PI. These changes not only provide evidence for the effectiveness of NOS blockade by the NO-clamp and the persistence of L-NAME within the fetal circulation until the end of the experiment, but they are also consistent with the idea that NOS blockade, even in the absence of SNP treatment, increased the sensitivity of the cardiac baroreflex. Further, a study in rats that compared the effects of circulating iron overload ( $20 \mathrm{mg}$ iron injected i.p. daily for $7 \mathrm{~d}$ followed by baroreflex evaluation) versus tissue iron overload $(10 \mathrm{mg}$ iron i.p. daily for $5 \mathrm{~d}$ followed by a resting period of $7 \mathrm{~d}$ and baroreflex evaluation) reported that baroreflex gain was only increased with tissue rather than serum iron overload (36). Since the entire experiment with the NO clamp in the present study lasted no more than $3 \mathrm{~h}$, it is also unlikely that the increase in fetal cardiac baroreflex sensitivity during NOS blockade is due to the nonspecific pharmacological effects of SNP.

One other possibility is that the enhanced baroreflex response in fetal life after NOS blockade in the present study is partly due to an effect of NOS inhibition at the level of the peripheral vasculature. Therefore, during NOS blockade, the increment in the pressor effects of phenylephrine will be greater and, hence, the cardiac baroreflex response will be enhanced. If this was the only effect of NOS blockade on the pressor and HR responses, one might expect an extension of the PI-ABP relationship rather than a change in its gradient. Since NOS blockade enhanced the slope of the relationship, a depressive effect of NO on baroreflex sensitivity is therefore strongly supported.

NO mediated inhibition of the cardiac baroreflex in fetal life has physiologic and clinical application. Wood et al. (37) reported that all three isoforms of NOS are constitutively expressed within the fetal brainstem, and that the expression of all three forms is reduced with advancing gestation. Wood et al. (37) speculated that the reduced expression of NOS in this brain region might play a role in the increased fetal baroreflex activity in late gestation. The data in the present study show that NOS inhibition augments baroreflex sensitivity, suggesting that NO indeed acts to reduce baroreflex sensitivity in fetal life, supporting this contention. Complicated pregnancy is usually associated with a reduction in fetal growth and small size at birth, which has been previously related to an increased susceptibility to hypertension in adult life (38). In turn, pregnancy complicated by preeclampsia, infection, or prolonged compression of the umbilical cord is related with an increase in fetal NO bioavailability $(39,40)$. If $\mathrm{NO}$ acts to diminish baroreflex sensitivity during fetal life, the offspring of complicated pregnancies may be predisposed to elevated resting $\mathrm{ABP}$ after birth. Therefore, investigation of changes in baroreflex sensitivity with the NO clamp technique at different stages of gestation and in particular toward term in normal and complicated pregnancies warrants further investigation to address the physiologic and clinical perspectives of the data shown in the present study.

Acknowledgments. We thank Mr. Scott Gentle and Mrs. Sue Nicholls for their invaluable help with the maintenance of the animals.

\section{REFERENCES}

1. Paintal AS 1973 Vagal sensory receptors and their reflex effects. Physiol Rev 53:159-227

2. Spyer KM 1981 Neural organisation and control of the baroreceptor reflex. Rev Physiol Biochem Pharmacol 88:24-124

3. Ismay MJ, Lumbers ER, Stevens AD 1979 The action of angiotensin II on the baroreflex response of the conscious ewe and the conscious fetus. J Physiol 288:467-479

4. Segar JL 1997 Ontogeny of the arterial and cardiopulmonary baroreflex during fetal and postnatal life. Am J Physiol 273:R457-R471

5. Tanaka K, Chiba T 1994 Nitric oxide synthase containing neurons in the carotid body and sinus of the guinea pig. Microsc Res Tech 29:90-93

6. Gai WP, Messenger JP, Yu YH, Gieroba ZJ, Blessing WW 1995 Nitric oxidesynthesising neurons in the central subnucleus of the nucleus tractus solitarius provide a major innervation of the rostral nucleus ambiguus in the rabbit. J Comp Neurol 357:348-361

7. Anderson CR, Edwards SL, Furness JB, Bredt DS, Snyder SH 1993 The distribution of nitric oxide synthase-containing autonomic preganglionic terminals in the rat. Brain Res 614:78-85

8. Lawrence AJ 1997 Nitric oxide as a modulator of medullary pathways. Clin Exp Pharmacol Physiol 24:760-763

9. Sener A, Smith FG 2001 Nitric oxide modulates arterial baroreflex control of heart rate in conscious lambs in an age-dependent manner. Am J Physiol Heart Circ Physiol 280:H2255-H2263 
10. Schroder HJ, Rybakowski C, Eisermann K, Tchirikov M, Ostermann S 2000 Unloading of baroreceptors by carotid occlusion does not increase heart rate in fetal sheep. Eur J Obstet Gynecol Reprod Biol 92:265-272

11. Walker AM, Cannata J, Dowling MH, Ritchie B, Maloney JE 1978 Sympathetic and parasympathetic control of heart rate in unanesthetized fetal and newborn lambs. Biol Neonate 33:135-143

12. Thakor AS, Giussani DA 2005 Calcitonin gene related peptide contributes to the umbilical haemodynamic defense response to acute hypoxaemia. J Physiol 536: 309-317

13. Gardner DS, Fowden AL, Giussani DA 2002 Adverse intrauterine conditions diminish the fetal defense against acute hypoxia by increasing nitric oxide activity. Circulation 106:2278-2283

14. Gardner DS, Giussani DA 2003 Enhanced umbilical blood flow during acute hypoxemia after chronic umbilical cord compression: a role for nitric oxide. Circulation 108:331-335

15. Dawes GS, Johnston BM, Walker DW 1980 Relationship of arterial pressure and heart rate in fetal, new-born and adult sheep. J Physiol 309:405-417

16. Chen RY, Fan FC, Schuessler GB, Chien S 1982 Baroreflex control of heart rate in humans during nitroprusside-induced hypotension. Am J Physiol 243:R18-R24

17. Minami N, Imai Y, Hashimoto J, Abe K 1995 The role of nitric oxide in the baroreceptor-cardiac reflex in conscious Wistar rats. Am J Physiol 269:H851-H855

18. Liu JL, Murakami H, Zucker IH 1996 Effects of NO on baroreflex control of heart rate and renal nerve activity in conscious rabbits. Am J Physiol 270:R1361-R1370

19. Ma R, Zhu GQ, Wang W 2005 Interaction of central Ang II and NO on the cardiac sympathetic afferent reflex in dogs. Auton Neurosci 118:51-60

20. Funakoshi K, Kadota T, Atobe Y, Nakano M, Goris RC, Kishida R 1999 Nitric oxide synthase in the glossopharyngeal and vagal afferent pathway of a teleost, Takifugu niphobles. The branchial vascular innervation. Cell Tissue Res 298:45-54

21. Lin LH, Cassell MD, Sandra A, Talman WT 1998 Direct evidence for nitric oxide synthase in vagal afferents to the nucleus tractus solitarii. Neuroscience 84:549-558

22. Chapleau MW 2004 Modulation of baroreflex function by altering inspiratory impedance: potential mechanisms and clinical implications. Clin Auton Res 14: 217-219

23. Ma SX, Fang Q, Morgan B, Ross MG, Chao CR 2003 Cardiovascular regulation and expressions of NO synthase-tyrosine hydroxylase in nucleus tractus solitarius of ovine fetus. Am J Physiol Heart Circ Physiol 284:H1057-H1063

24. Paton JF, Deuchars J, Ahmad Z, Wong LF, Murphy D, Kasparov S 2001 Adenoviral vector demonstrates that angiotensin II-induced depression of the cardiac baroreflex is mediated by endothelial nitric oxide synthase in the nucleus tractus solitarii of the rat. J Physiol 531:445-458

25. Waki H, Kasparov S, Wong LF, Murphy D, Shimizu T, Paton JF 2003 Chronic inhibition of endothelial nitric oxide synthase activity in nucleus tractus solitarii enhances baroreceptor reflex in conscious rats. J Physiol 546:233-242
26. Pontieri V, Venezuela MK, Scavone C, Michelini LC 1998 Role of endogenous nitric oxide in the nucleus tratus solitarii on baroreflex control of heart rate in spontaneously hypertensive rats. J Hypertens 16:1993-1999

27. Smith SA, Mitchell JH, Li J 2004 Independent modification of baroreceptor and exercise pressor reflex function by nitric oxide in the nucleus tractus solitarius. Am J Physiol Heart Circ Physiol 288:H2068-H2076

28. Chowdhary S, Townend JN 1999 Role of nitric oxide in the regulation of cardiovascular autonomic control. Clin Sci (Lond) 97:5-17

29. Di Paola ED, Vidal MJ, Nistico G 1991 L-Glutamate evokes the release of endothelium-derived relaxing factor-like substance from the rat nucleus tractus solitarius. J Cardiovasc Pharmacol 17:S269-S272

30. Ma S, Abboud FM, Felder RB 1995 Effects of L-arginine-derived nitric oxide synthesis on neuronal activity in nucleus tractus solitarius. Am J Physiol 268:R487R491

31. Tagawa T, Imaizumi T, Harada S, Endo T, Shiramoto M, Hirooka Y, Takeshita A 1994 Nitric oxide influences neuronal activity in the nucleus tractus solitarius of rat brainstem slices. Circ Res 75:70-76

32. Balligand JL, Kelly RA, Marsden PA, Smith TW, Michel T 1993 Control of cardiac muscle cell function by an endogenous nitric oxide signaling system. Proc Natl Acad Sci U S A 90:347-351

33. Conlon K, Collins T, Kidd C 1996 Modulation of vagal actions on heart rate produced by inhibition of nitric oxide synthase in the anaesthetized ferret. Exp Physiol 81:547-550

34. Sears CE, Choate JK, Paterson DJ 1999 NO-cGMP pathway accentuates the decrease in heart rate caused by cardiac vagal nerve stimulation. J Appl Physiol $86: 510-516$

35. Roncaroli F, van Eldik R, Olabe JA 2005 Release of NO from reduced nitroprusside ion. Iron-dinitrosyl formation and NO-disproportionation reactions. Inorg Chem 44:2781-2790

36. Cardoso LM, Pedrosa ML, Silva ME, Moraes MF, Colombari E, Chianca DA Jr 2005 Baroreflex function in conscious rats submitted to iron overload. Braz J Med Biol Res 38:205-214

37. Wood CE, Chen G, Keller-Wood M 2005 Expression of nitric oxide synthase isoforms is reduced in late-gestation ovine fetal brainstem. Am J Physiol Regul Integr Comp Physiol 289:R613-R619

38. Barker DJ 1998 Mothers, Babies, and Disease in Later Life. Edinburgh: Churchill Livingstone

39. Norris LA, Higgins JR, Darling MR, Walshe JJ, Bonnar J 1999 Nitric oxide in the uteroplacental, fetoplacental, and peripheral circulations in preeclampsia. Obstet Gynecol 93:958-963

40. von Mandach U, Lauth D, Huch R 2003 Maternal and fetal nitric oxide production in normal and abnormal pregnancy. J Matern Fetal Neonatal Med 13:22-27 